\title{
Magnetic resonant $x$-ray diffraction study of europium telluride
}

\author{
B. Díaz, ${ }^{1, *}$ E. Granado, ${ }^{2,3}$ E. Abramof, ${ }^{1}$ P. H. O. Rappl, ${ }^{1}$ V. A. Chitta, ${ }^{4}$ and A. B. Henriques ${ }^{4}$ \\ ${ }^{1}$ Laboratório Associado de Sensores e Materiais, Instituto Nacional de Pesquisas Espaciais, CP515, 12245-970 São José dos Campos, \\ SP, Brazil \\ ${ }^{2}$ Instituto de Física “Gleb Wataghin,” UNICAMP, 13083-970 Campinas, SP, Brazil \\ ${ }^{3}$ Laboratório Nacional de Luz Sincrotron, CP6192, 13084-971 Campinas, SP, Brazil \\ ${ }^{4}$ Instituto de Física, Universidade de São Paulo, CP66318, 05315-970 São Paulo, SP, Brazil \\ (Received 25 June 2008; revised manuscript received 27 September 2008; published 27 October 2008)
}

\begin{abstract}
Here we use magnetic resonant $\mathrm{x}$-ray diffraction to study the magnetic order in a $1.5 \mu \mathrm{m}$ EuTe film grown on (111) $\mathrm{BaF}_{2}$ by molecular-beam epitaxy. At Eu $L_{\mathrm{II}}$ and $L_{\mathrm{III}}$ absorption edges, a resonant enhancement of more than two orders was observed for the $\sigma \rightarrow \pi^{\prime}$ diffracted intensity at half-order reciprocal-lattice points, consistent with the magnetic character of the scattering. We studied the evolution of the $\left(\frac{1}{2} \frac{1}{2} \frac{1}{2}\right)$ magnetic reflection with temperature. When heating toward the Neel temperature $\left(T_{N}\right)$, the integrated intensity decreased monotonously and showed no hysteresis upon cooling again, indicating a second-order phase transition. A power-law fit to the magnetization versus temperature curve yielded $T_{N}=9.99(1) \mathrm{K}$ and a critical exponent $\beta=0.36(1)$, which agrees with the renormalization theory results for three-dimensional Heisenberg magnets. The fits to the sublattice magnetization dependence with temperature, disregarding and considering fourth-order exchange interactions, evidenced the importance of the latter for a correct description of magnetism in EuTe. A value of 0.009 was found for the $\left(2 j_{1}+j_{2}\right) / J_{2}$ ratio between the Heisenberg $J_{2}$ and fourth-order $j_{1,2}$ exchange constants. The magnetization curve exhibited a round-shaped region just near $T_{N}$ accompanied by an increase in the magnetic peak width, which was attributed to critical scattering above $T_{N}$. The comparison of the intensity ratio between the $\left(\frac{1}{2} \frac{1}{2} \frac{1}{2}\right)$ and the $\left(1 \frac{1}{2} 1 \frac{1}{2} 1 \frac{1}{2}\right)$ magnetic reflections proved that the $\mathrm{Eu}^{2+}$ spins align within the (111) planes, and the azimuthal dependence of the $\left(\frac{1}{2} \frac{1}{2} \frac{1}{2}\right)$ magnetic peak is consistent with the model of equally populated $\mathrm{S}$ domains.
\end{abstract}

DOI: 10.1103/PhysRevB.78.134423

PACS number(s): 75.25.+z, 75.40.Cx, 75.60.Ej, 75.10.Dg

\section{INTRODUCTION}

Resonant $\mathrm{x}$-ray diffraction is an useful technique for the study of magnetic materials. ${ }^{1}$ The intrinsic weakness of magnetic relative to charge diffraction intensities is partially overthrown in resonant diffraction with the strong intensity enhancement occurring at core absorption edges. ${ }^{2}$ The availability of very intense white synchrotron sources has been fundamental for the development of this technique. Compared to well established neutron diffraction, magnetic x-ray diffraction has the advantage of a higher resolution inherent to synchrotron sources, useful, for example, in the study of magnetoelastic effects accompanying phase transitions ${ }^{3}$ and phase coexistence phenomena. ${ }^{4}$

The magnetic properties of europium chalcogenides $\mathrm{Eu} X$ $(X=\mathrm{O}, \mathrm{S}, \mathrm{Se}, \mathrm{Te})$ have been extensively studied because of the strong localized moments which make them classical Heisenberg magnets. Their magnetic properties arise from the half-filled $4 f$ shells of $\mathrm{Eu}^{2+}$ cations. They have $\mathrm{NaCl}$ crystal structure, and their magnetic ordering depends on the anion, which affects the lattice parameter, the crystal field, and ultimately the nearest-neighbors $J_{1}$ and next-nearestneighbors $J_{2}$ Heisenberg exchange constants. ${ }^{5}$ The last member of this series, EuTe, has a type-II, MnO, antiferromagnetic (AFM) structure below the Neel temperature $T_{N}=9.6 \mathrm{~K}$ for the bulk material. ${ }^{6}$ Early studies demonstrated that the sublattice magnetization lies in the (111) planes. The spins are parallel within the (111) planes and antiparallel with those spins in adjacent (111) planes. The four equivalent (111), (-111), (1-11), and (11-1) planes result in the for- mation of the so-called $T$ domains. Additional in-plane anisotropy favors spin alignment in any of the three equivalent $\langle 11-2\rangle$ azimuths, forming the $S$ domains. ${ }^{7}$

Below $T_{N}$, the AFM ordering of EuTe provides a straightforward separation between charge and magnetic diffraction peaks. In a very recent work, we used resonant x-ray diffraction to study the magnetic order in EuTe/PbTe multilayers. ${ }^{8}$ We were able to measure the magnetic diffraction signal from a 40 monolayers EuTe film and the interlayer correlations among the individual EuTe layers in a EuTe/PbTe superlattice. In this work, we present a detailed study of the magnetic ordering of a $1.5 \mu \mathrm{m}$ EuTe epitaxial film as a function of temperature using resonant x-ray diffraction. We investigate the resonant behavior at the $\mathrm{Eu} L_{\mathrm{II}}$ and $L_{\mathrm{III}} \mathrm{ab}-$ sorption edges, where the magnetically diffracted intensity was found to increase by more than two orders. The high counting rates at resonance allowed to study the evolution of the magnetic peak with temperature, even close to $T_{N}$, obtaining information about the magnetic transition order, temperature, and critical exponent $\beta$. The fits to the temperature dependence of the sublattice magnetization showed the importance of fourth-order interactions for a proper description of magnetism in EuTe.

\section{EXPERIMENT}

An EuTe film of $1.5 \mu \mathrm{m}$ was grown on (111) oriented $\mathrm{BaF}_{2}$ substrate in a Riber 32P molecular-beam epitaxy (MBE) system equipped with $\mathrm{Eu}, \mathrm{Te}$, and $\mathrm{BaF}_{2}$ sources. The freshly cleaved $\mathrm{BaF}_{2}$ substrate was preheated to $400{ }^{\circ} \mathrm{C}$ for 
$15 \mathrm{~min}$ and was kept rotating during the growth to ensure maximum uniformity. A thin $\mathrm{BaF}_{2}$ protective layer was grown on top of the EuTe film to avoid oxidation. The rocking curve measured with a Philips X'Pert MRD diffractometer around the (222) EuTe Bragg peak exhibited a full width at half maximum (FWHM) of 300", indicating high crystalline quality.

The resonant $\mathrm{X}$-ray measurements were made at the XRD2 beamline, placed after a dipolar source, at the Brazilian Synchrotron Facility. ${ }^{9}$ The beam was vertically focused with a bent Rh-coated mirror, which also filtered higherorder harmonics. A double-bounce (111) Si monochromator was placed after the $\mathrm{Rh}$ mirror, achieving an energy resolution of $5 \mathrm{eV}$. The beam is focused at the sample position in a spot of $2 \mathrm{~mm}$ (horizontal) $\times 0.6 \mathrm{~mm}$ (vertical), with a flux of the order of $10^{10}$ photons/s. The sample was mounted in a cryostat, which was itself mounted onto the Eulerian cradle of a Huber $4+2$ circle diffractometer, with vertical scattering plane. The cryostat is a closed cycle cryorefrigerator ARS Displex DE-202, whose last stage consists of an open cycle Joule-Thompson circuit. The system achieves a base temperature of $1.6 \mathrm{~K}$, with stability of $\pm 0.001 \mathrm{~K}$. Three beryllium domes separate the sample in the cryostat vacuum from the atmosphere. A scintillation detector was used in all experiments except for the measurements of the magnetic peak for different azimuths, where a solid-state Canberra detector was employed.

A commercial superconducting quantum interference device (SQUID) magnetometer MPMS-5 from Quantum Design was used for the magnetization measurements.

\section{RESULTS AND DISCUSSION}

\section{A. Resonant behavior at Eu $L_{\mathrm{II}}$ and $L_{\mathrm{III}}$ absorption edges}

Half-order peaks, with $h k l\left(\frac{1}{2} \frac{1}{2} \frac{1}{2}\right),\left(1 \frac{1}{2} 1 \frac{1}{2} 1 \frac{1}{2}\right)$, etc., appeared when the sample was cooled below the Neel temperature. To test the magnetic origin of the half-order reflections we used a graphite analyzer. The reflections (003) and (001) of the analyzer corresponded to scattering angles $2 \theta$ of $93.3^{\circ}$ and $27.96^{\circ}$, respectively, for the wavelength $1.6289 \AA$ of the $\mathrm{Eu} L_{\mathrm{II}}$ edge. The intensity of $\sigma$-polarized radiation incident on the analyzer will reduce according to the reflecting power of each analyzer reflection, while that of $\pi$-polarized incident radiation will suffer additional weakening proportional to $\cos ^{2}(2 \theta){ }^{1}$ Hence, the (003) reflection selects the $\sigma \rightarrow \sigma^{\prime}$ scattering from the sample, while the (001) reflection does not discriminate $\sigma \rightarrow \sigma^{\prime}$ from $\sigma \rightarrow \pi^{\prime}$ scattering channels. The intensity ratio of the $\sigma \rightarrow \sigma^{\prime}$ (222) EuTe charge reflection for the (003) and (001) analyzer reflections was 0.204. Meanwhile, the intensity ratio of the $\left(\frac{1}{2} \frac{1}{2} \frac{1}{2}\right)$ EuTe reflection was only 0.012 , signaling nearly pure $\sigma \rightarrow \pi^{\prime}$ scattering, consistent with the magnetic origin of the reflection in a dipolar resonance.

Sweeping the energy across the Eu $L_{\mathrm{II}}$ and $L_{\mathrm{III}}$ edges resulted in a strong intensity enhancement of the EuTe halforder magnetic reflections in more than 2 orders of magnitude, as shown in Fig. 1 for the $\left(1 \frac{1}{2} 1 \frac{1}{2} 1 \frac{1}{2}\right)$ magnetic peak. The measurements were made at $1.6 \mathrm{~K}$ and later corrected

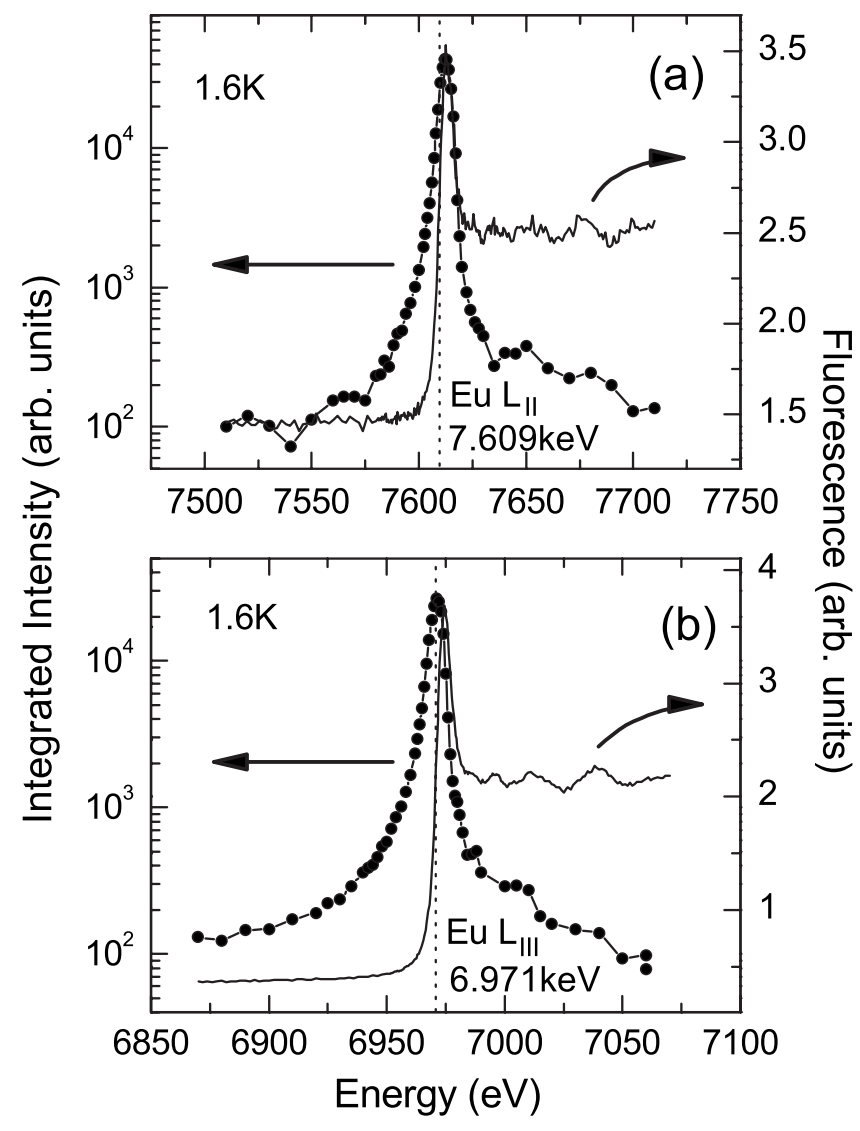

FIG. 1. Energy dependence of the $\left(1 \frac{1}{2} 1 \frac{1}{2} 1 \frac{1}{2}\right)$ EuTe magnetic peak integrated intensity, corrected for absorption, and fluorescence, measured around Eu $L_{\mathrm{II}}$ (a) and $L_{\mathrm{III}}$ (b) absorption edges.

for self-absorption. The fluorescence yield was also measured as a function of energy. The Eu $L_{\mathrm{II}}$ and $L_{\mathrm{III}}$ absorption edges, determined as the maximum value of the fluorescence first derivative, were at 7609.5 and $6970.9 \mathrm{eV}$, respectively. Maximum intensity enhancements of the magnetic reflections occurred at $7612 \mathrm{eV}$ for the $L_{\mathrm{II}}$ and at $6971 \mathrm{eV}$ for the $L_{\text {III }}$ Eu absorption edges. Squared Lorentzian fits to the resonance curves yielded resonance widths of 6.2 and $5.6 \mathrm{eV}$ for the $L_{\mathrm{II}}$ and $L_{\mathrm{III}}$ absorption edges, respectively, after deconvolution with the beam energy width.

The resonant enhancements over two orders observed here at $L_{\mathrm{II}}$ and $L_{\mathrm{III}}$ edges of $\mathrm{Eu}$ can be compared to those of gadolinium in a study of $\mathrm{Gd}_{2} \mathrm{IrIn}_{8}$, where an intensity increase of over 3 orders of magnitude was observed at the same absorption edges. ${ }^{2}$ The resonant enhancement depends on the magnetic polarization of the rare-earth $5 d$ electronic levels involved in the dipolar transition $2 p \rightarrow 5 d$. The polarization occurs by hybridization with $4 f$ magnetic electrons, and in $\mathrm{Gd}_{2} \operatorname{IrIn}_{8}$ it occurs via In $5 p$ electrons, or on-site Gd $4 f-5 d$, since the $\mathrm{Gd}$ ions occupy noncentrosymmetric sites. ${ }^{2}$ For EuTe, the centrosymmetric structure does not allow on-site hybridization, and the polarization would occur only through the neighboring Eu and Te atoms, which may explain the smaller resonances observed.

\section{B. Temperature dependence of magnetic EuTe Bragg peaks}

In order to investigate the transition to the AFM ordered state, we measured $\theta / 2 \theta$ scans along the $\left(\frac{1}{2} \frac{1}{2} \frac{1}{2}\right)$ magnetic 


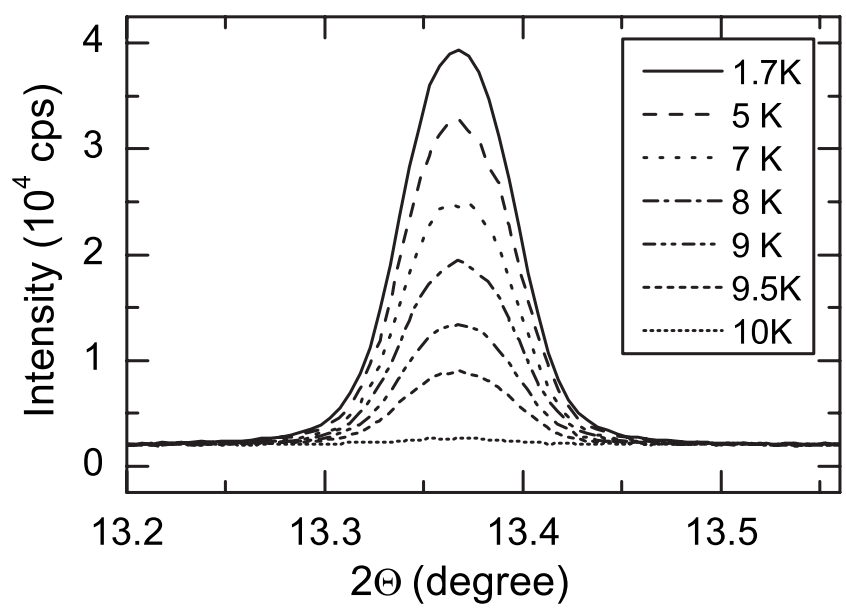

FIG. 2. $\theta / 2 \theta$ scans along the $\left(\frac{1}{2} \frac{1}{2} \frac{1}{2}\right)$ magnetic reflection for different temperatures, measured at $\mathrm{Eu} L_{\mathrm{III}}$ edge.

peak for different temperatures between 1.7 and $11 \mathrm{~K}$ (Fig. 2). The peak shape was always symmetric, denoting no significant strain gradient. During these measurements, the intensity was normalized with a monitor detector placed before the sample to compensate for the steady decrease of the beam flux after injection. High counting rates, up to 39000 counts/s for the lowest temperature (Fig. 2), proved both the sample quality and the suitability of the technique to analyze EuTe thin layers.

Figure 3(a) shows the temperature dependence of the normalized sublattice magnetization obtained from the square root of the magnetic peak integrated intensity. The magnetization decreases continuously to zero as the transition temperature is approached, indicating a second-order phase transition. Measurements of the magnetic peak during heating and cooling showed no hysteresis, also signaling a secondorder phase transition. The phase transitions for both $\mathrm{MnO}$ and EuTe in the unstrained state are expected to be of first order within renormalization theory. ${ }^{10,11}$ The presence of strain above a certain threshold value should be able to induce a second-order phase transition beyond a tricritical point, which has been demonstrated for $\mathrm{MnO} .^{12}$ The presence of biaxial strain in our EuTe film does not allow us to draw any conclusions on the transition order of unstrained EuTe. However, it must be stressed that first-order phase transitions were also predicted for materials such as $\mathrm{CeS}$, $\mathrm{CeSe}$, and $\mathrm{CeTe}$, whose transitions appear continuous within experimental accuracy. ${ }^{10}$

To obtain the transition temperature $T_{N}$ and the critical exponent $\beta$, the sublattice magnetization versus temperature data were fitted with a power law in the region $0.1>t$ $>0.006$, where $t=\left(T_{N}-T\right) / T_{N}$ is the reduced temperature [Fig. 3(a) inset]. The fit yielded $T_{N}=9.99(1) \mathrm{K}$ and $\beta=0.36(1)$. The same results are obtained when reducing the fitting range to $0.05>t>0.006$. The upper temperature boundary of the fitting interval leaves a narrow round-shaped region of the magnetization curve near $T_{N}$ outside of the fit. In the rounded region, an increase in the magnetic peak width was observed as the temperature increased [Fig. 3(b)]. The transition temperature obtained coincides with that found from magnetization versus temperature SQUID mea-

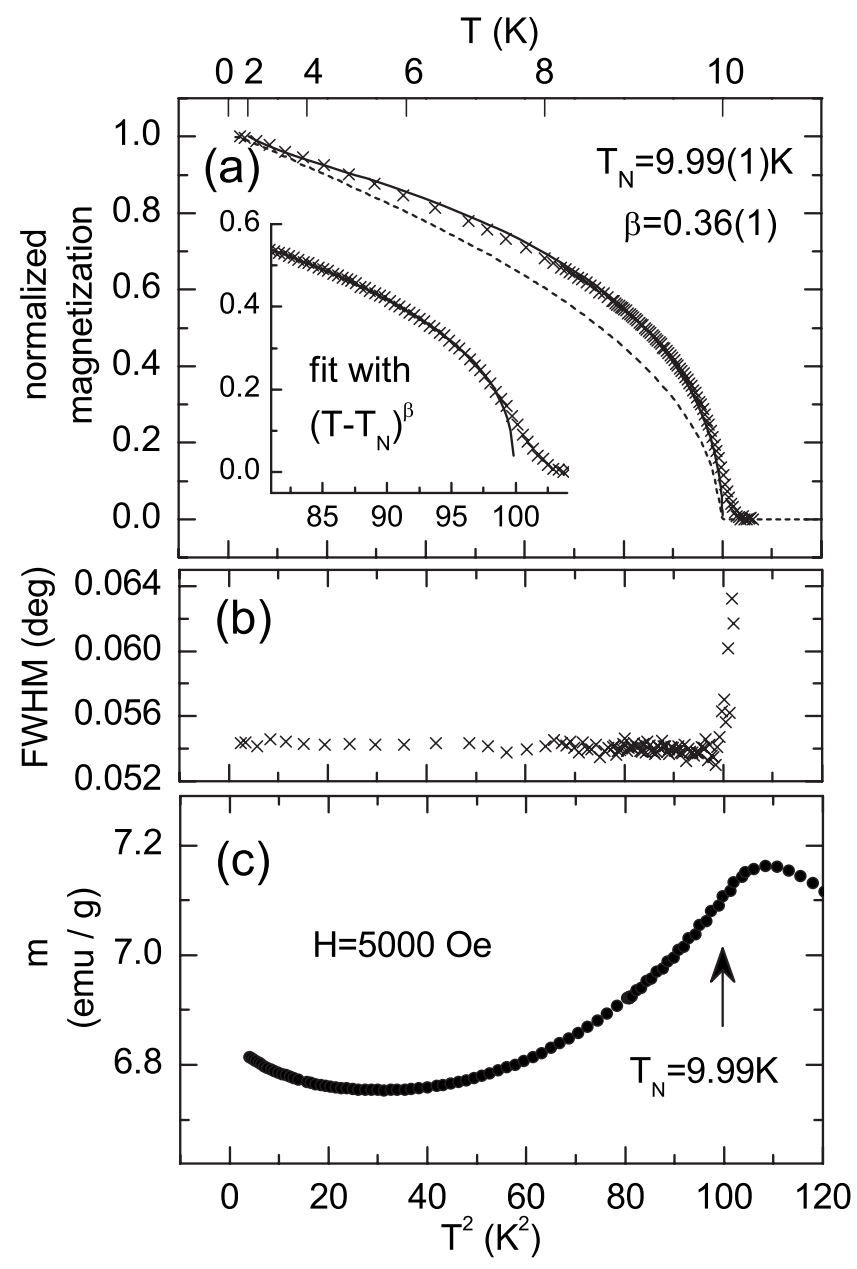

FIG. 3. Normalized sublattice magnetization, obtained from the square root of the $\left(\frac{1}{2} \frac{1}{2} \frac{1}{2}\right)$ magnetic peak integrated intensity, is shown in (a) as a function of the squared temperature along with theoretical models disregarding (dotted line) and considering (solid line) fourth-order interactions. The inset magnifies the region closest to the Neel temperature and shows a power-law fit (solid line) from which the critical exponent $\beta$ and $T_{N}$ were obtained. The magnetic peak full width at half maximum is shown in (b). The $T_{N}$ obtained from SQUID magnetization measurements shown in (c) agrees with diffraction results.

surements [Fig. 3(c)], where $T_{N}$ was taken as the point where $M$ varied the most. ${ }^{6}$

The rounded region of the magnetization curve can be due to critical scattering above $T_{N}$, as suggested by the increasing peak width within this region [Fig. 3(b)], or to strain inhomogeneities within the sample. For EuTe, next-nearestneighbor exchange constant $J_{2}$ and the ordering temperature $T_{N}$ are only slightly dependent on lattice parameter. ${ }^{13}$ Strain inhomogeneities able to produce a spread on $T_{N}$ over $\sim 0.15 \mathrm{~K}$ would also produce peaks about 10 times wider than observed here. Besides, if the rounded shape was a result of strain inhomogeneities, in the vicinity of $T_{N}$ only the more strained regions, still having magnetic order, would be able to scatter, leading to a shift of the peak to lower $\theta$ as $T$ increases, ${ }^{12}$ which is also not observed. The rounded shape of the magnetization curve around $T_{N}$ is then ascribed to critical scattering above $T_{N}$. 
TABLE I. Values of $a^{*}=d \sqrt{h^{2}+k^{2}+l^{2}}$ for different $h k l$ Bragg reflections measured at $15 \mathrm{~K}$. The offset $(\alpha)$ and $\mathrm{x}$-ray penetration depth $(z)$ are also given for each reflection.

\begin{tabular}{lccc}
\hline \hline$h k l$ & $\begin{array}{c}a^{*}=d \sqrt{h^{2}+k^{2}+l^{2}} \\
(\AA)\end{array}$ & $\begin{array}{c}\alpha \\
(\mathrm{deg})\end{array}$ & $\begin{array}{c}z \\
(\mu \mathrm{m})\end{array}$ \\
\hline 111 & 6.5874 & 0 & 0.43 \\
222 & 6.5875 & 0 & 0.86 \\
224 & 6.5849 & 19.47 & 0.97 \\
313 & 6.5843 & 22.00 & 1.00 \\
202 & 6.5797 & 35.26 & 0.34 \\
115 & 6.5786 & 38.94 & 0.33 \\
024 & 6.5781 & 39.23 & 0.22 \\
$3-11$ & 6.5726 & 58.52 & 0.83 \\
\hline \hline
\end{tabular}

To obtain more information on the strain state of the sample, we measured at $15 \mathrm{~K}$ several symmetric and asymmetric structural reflections, with different offsets $\alpha$ relative to the normal direction and hence different penetration depths (Table I). The lattice parameters obtained from these reflections nearly lie in a straight line when plotted against $\sin ^{2} \alpha$, denoting a homogeneous in-depth strain. Also, the symmetric peak profiles (Fig. 2) indicate homogeneous indepth strain. This contrasts with thicker films grown on closer lattice matched PbTe buffers on $\mathrm{BaF}_{2}$ substrates, which exhibit clear asymmetric peaks resulting from strain profiles. ${ }^{14}$ Our EuTe film was grown directly on a $\mathrm{BaF}_{2}$ substrate, and the initial island growth mode that creates a highly defective narrow region before the layer-by-layer growth proceeds must be the reason for the homogeneous in-depth strain.

The value found for $T_{N}$, of $9.99 \mathrm{~K}$, from the power-law fit [Fig. 3(a) inset], is slightly higher than the bulk value, of $9.6 \mathrm{~K}^{6}$ Small variations of $T_{N}$ in thin EuTe films have already been observed before. ${ }^{14}$ This can be due to the strain present at the EuTe layer. From the mean-field theory we know that $T_{N}=-4 J_{2} S(S+1) / k_{b},{ }^{5,13}$ where $k_{b}$ is the Boltzmann constant and $S=7 / 2$ is the spin. Hence, a change in $T_{N}$ is expected if $J_{2}$ varies for any reason. Previous studies of bulk EuTe at high pressures found a slight increase in $\left|J_{2}\right|$ for smaller lattice parameters. ${ }^{13}$ The situation is more complicated in our EuTe thin film, with biaxial strain compressive in the plane and tensile in the [111] direction normal to the film plane. From simple geometrical and elasticity considerations it can be seen that the net effect of this biaxial strain for EuTe is a smaller cation-anion distance. A smaller Eu-Te distance is expected to favor $J_{2}$ superexchange constant, and hence increase $T_{N}$, as observed here.

The value found for the critical exponent $\beta=0.36(1)$ coincides with the prediction for a three-dimensional (3D) Heisenberg (isotropic) magnet obtained from renormalization theory. ${ }^{15}$ In a previous study of EuTe, an anomalous behavior for the specific-heat critical exponent of EuTe was found. ${ }^{16}$ The critical exponent showed a crossover at $|t|$ $\sim 0.01$, with a value higher (smaller) for $|t|<0.01 \quad(|t|$ $>0.01)$ than that predicted by the renormalization theory. The hypothesis was placed that this could be due to the an- isotropy arising from the magnetic dipole-dipole interaction, or by the occurrence of a first-order transition, ${ }^{16}$ which would be consequent with the existence of a tricritical point for EuTe.

Anisotropic fourth-order interactions could also explain the anomalies of the specific heat. In fact, it was suggested that EuTe has important fourth-order interactions very influent on the spin dynamics. ${ }^{17,18}$ Here, we calculated the temperature dependence of the sublattice magnetization following the approach in Ref. 19, considering the Hamiltonian $J \mathbf{S}_{i} \cdot \mathbf{S}_{j}-j\left(\mathbf{S}_{i} \cdot \mathbf{S}_{j}\right)^{2}$, where the first term describes the wellknown isotropic Heisenberg interactions and the second term stands for fourth-order interactions. Disregarding strain energy, the internal energy of the system is expressed as the exchange energy

$$
U_{\text {exch }}=-\frac{1}{2} N\left[z_{2} J_{2}(\sigma S)^{2}+z_{2} j_{2}(\sigma S)^{4}+z_{1} j_{1}(\sigma S)^{4}\right],
$$

where $N$ is the number of spins, $z_{1,2}$ are the number of nearest and next-nearest neighbors, $\sigma$ is the relative magnetization, and $S=7 / 2$ is the pure spin magnetic moment of Eu atoms. The frustration of one half of the nearest neighbors cancels the term linear in $J_{1}$. Considering that the free-energy $U-T S^{*}$ derivative relative to $\sigma$ at constant temperature must be zero at equilibrium, one obtains

$$
\frac{T}{T_{N}}=-\frac{3 N k_{b} S \sigma}{(S+1)\left(\frac{\partial S^{*}}{\partial \sigma}\right)_{T}}\left[1+2 S^{2} \sigma^{2}\left(2 j_{1}+j_{2}\right) / J_{2}\right]
$$

where $S^{*}$ is the spin entropy, and was derived calculating the first 12 coefficients of the power-series expansion, with the method described in Ref. 20.

Equation (2) leads to the Brillouin function when fourthorder interactions are neglected. The experimental magnetization versus temperature dependence in Fig. 3(a) was fitted considering and disregarding fourth-order interactions (solid and dotted lines, respectively). It can be seen that only the model including fourth-order interactions properly reproduces the experiment. This fit yielded a value 0.009 for the quotient $\left(2 j_{1}+j_{2}\right) / J_{2}$ between the fourth-order and secondorder exchange constants, smaller than the previously reported value of $0.016 .{ }^{18}$ However, the Neel temperature of $7.8 \mathrm{~K}$ reported in Ref. 18 is also rather far from the usually reported value of $9.6 \mathrm{~K},{ }^{6}$ which likely results from stoichiometry problems that might have affected their exchange constants quotient. The value for the quotient $\left(2 j_{1}+j_{2}\right) / J_{2}$ found here must also be affected by the presence of biaxial strain. Unfortunately, the strain influence could not be separated since the dependence of the EuTe exchange constants on interatomic distances is not well known.

The abscissa axis of Fig. 3(a) is linear in $T^{2}$, instead of $T$, to show that the magnetization nearly follows a $T^{2}$ law up to $\sim 0.8 T_{N}$. This contrasts with the $T^{3 / 2}$ law predicted by Bloch. Many ferromagnetic (FM) and AFM materials also follow this $T^{2}$ law over wide temperature ranges, a behavior that has been attributed to fourth-order interactions. ${ }^{17,21}$ 
The relative importance of fourth-order interactions in EuTe can be understood reminding that half the nearest neighbors are frustrated. In EuTe, the nearest-neighbor exchange constant $J_{1}$ is positive, favoring a ferromagnetic alignment. But, from the 12 nearest neighbors, only the six on the same (111) plane are parallelly aligned. The other six nearest neighbors, half in each adjacent (111) planes, are antiparallel (frustrated). This cancels the nearest neighbors $J_{1} \mathbf{S}_{i} \cdot \mathbf{S}_{j}$ term on the Hamiltonian, favoring the influence of fourth-order interactions. This term reappears weakly in the presence of a rhombohedral distortion due to the $J_{1}$ dependence on $\mathrm{Eu}-\mathrm{Eu}$ distance.

\section{Magnetic moments orientation within the lattice}

To a first approximation, the intensity of the x-ray magnetic diffraction depends on the squared scalar product between the scattered wave vector and the magnetic moment. Hence, a comparison of the intensity of different magnetic reflections can give insight on the magnetic-moment directions within the lattice. To achieve this, we measured $\theta$ and $\theta / 2 \theta$ scans around the $\left(\frac{1}{2} \frac{1}{2} \frac{1}{2}\right)$ and the $\left(1 \frac{1}{2} 1 \frac{1}{2} 1 \frac{1}{2}\right)$ magnetic reflections. The integrated intensity of each reflection was found integrating inside the revolution ellipsoid, whose intensity profiles along the $x, y$, and $z$ axes were the $\theta, \theta$, and $\theta / 2 \theta$ scans, respectively. The result for the intensity ratio of $\left(\frac{1}{2} \frac{1}{2} \frac{1}{2}\right)$ and $\left(1 \frac{1}{2} 1 \frac{1}{2} 1 \frac{1}{2}\right)$ magnetic reflections was 3.4(1). Considering the magnetic moments all aligned in the same inplane direction and correcting for different path lengths of the beam on each case, the expected intensity ratio is 3.38 , which is fairly equal to the experimental value.

It can be easily proved that the same theoretical intensity ratio is obtained for any direction of the magnetic moments within the plane, except for the two particular cases where the magnetic moment is perpendicular to the scattered wave vector, when the intensity falls to zero. Also, the same intensity ratio is expected if we consider that the three possible $S$ domains are equally populated. Therefore, the previous method allows concluding that the spins lie in the plane of the film, but does not answer the question of whether they are all parallel or distributed in the three possible $S$ domains. To clarify this, we measured the intensity of the $\left(\frac{1}{2} \frac{1}{2} \frac{1}{2}\right)$ magnetic peak in different azimuths (Fig. 4). If only one $S$ domain is populated, a cosine-like dependence of the intensity with the azimuth is expected (solid line in Fig. 4). When the three $S$ domains are equally populated, the intensity is expected to remain constant for any azimuth, which roughly coincides with our experimental result (Fig. 4). We can then conclude that the three possible $S$ domains are approximately equally populated.

\section{CONCLUSIONS}

In this work we studied the magnetic ordering of EuTe in a vicinity of the Neel temperature $T_{N}$ using magnetic x-ray

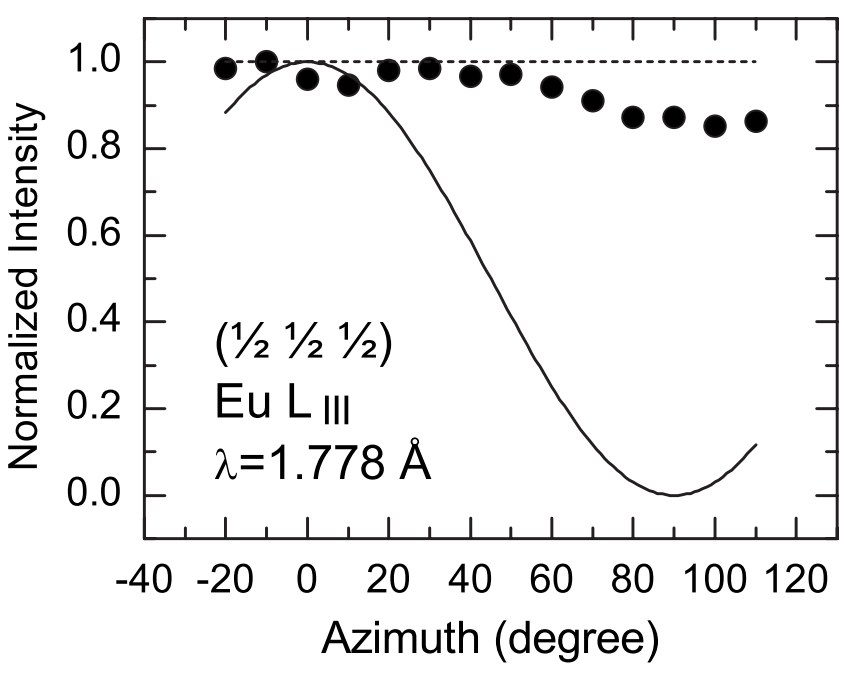

FIG. 4. Integrated intensity of $\theta / 2 \theta$ scans along the $\left(\frac{1}{2} \frac{1}{2} \frac{1}{2}\right)$ magnetic reflection for different azimuths (circles). The solid line is the theoretical intensity behavior if only one $S$ domain is populated, while the dotted line is the expectation when the three $S$ domains are equally populated.

diffraction. Below $T_{N}$ half-order peaks appear with pure $\sigma \rightarrow \pi^{\prime}$ scattering, which proves their magnetic origin. Strong intensity enhancements of more than 2 orders of magnitude were observed both in $L_{\mathrm{II}}$ and $L_{\mathrm{III}} \mathrm{Eu}$ absorption edges. We studied the evolution of the $\left(\frac{1}{2} \frac{1}{2} \frac{1}{2}\right)$ magnetic reflection near the Neel temperature. When heating toward $T_{N}$, the integrated intensity decreases monotonously and shows no hysteresis upon cooling again, indicating a second-order phase transition. The critical exponent $\beta=0.36(1)$ agrees with renormalization theory results for 3D Heisenberg magnets. The magnetization dependence with temperature could only be successfully reproduced with a model that included fourth-order interactions, yielding a value of 0.009 for the $\left(2 j_{1}+j_{2}\right) / J_{2}$ ratio. The wide range over which the magnetization behaved linearly with $T^{2}$ also supports the importance of quadratic interactions. The comparison of the intensity ratio of two magnetic reflections is consistent with the model in which the $\mathrm{Eu}^{2+}$ spins align within the (111) planes on equally populated $S$ domains.

\section{ACKNOWLEDGMENTS}

We thank G. Kellerman and C. Azimonte for technical assistance and $\mathrm{CNPq}$ (Contract No. 142292/2004-4) and FAPESP (Contract No. 2005/05194-1) for financial support. 
*bdiazmoreno@gmail.com

${ }^{1}$ S. W. Lovesey and S. P. Collins, X-Ray Scattering and Absorption by Magnetic Materials (Oxford University Press, New York, 1996).

${ }^{2}$ E. Granado, P. G. Pagliuso, C. Giles, R. Lora-Serrano, F. Yokaichiya, and J. L. Sarrao, Phys. Rev. B 69, 144411 (2004).

${ }^{3}$ T. Chatterji, K. D. Liß, T. Tschentscher, B. Janossy, J. Strempfer, and T. Brückel, Solid State Commun. 131, 713 (2004).

${ }^{4}$ Y. Narumi, K. Katsumata, T. Nakamura, Y. Tanaka, S. Shimomura, T. Ishikawa, and M. Yabashi, J. Phys.: Condens. Matter 16, L57 (2004).

${ }^{5}$ I. N. Goncharenko and I. Mirebeau, Phys. Rev. Lett. 80, 1082 (1998).

${ }^{6}$ N. F. Oliveira, S. Foner, Y. Shapira, and T. B. Reed, Phys. Rev. B 5, 2634 (1972).

${ }^{7}$ J. W. Battles and G. E. Everett, Phys. Rev. B 1, 3021 (1970).

${ }^{8}$ B. Diaz, E. Granado, E. Abramof, P. H. O. Rappl, V. A. Chitta, and A. B. Henriques, Appl. Phys. Lett. 92, 242511 (2008).

${ }^{9}$ C. Giles, F. Yokaichiya, S. W. Kycia, L. C. Sampaio, D. C. Ardiles-Saravia, M. K. K. Franco, and R. T. Neuenschwander, J. Synchrotron Radiat. 10, 430 (2003).

${ }^{10}$ D. Mukamel and D. J. Wallace, J. Phys. C 12, L851 (1979).
${ }^{11}$ W. R. Johanson and D. C. McCollum, Phys. Rev. B 22, 2435 (1980).

${ }^{12}$ D. Bloch, D. Hermann-Ronzaud, C. Vettier, W. B. Yelon, and R. Alben, Phys. Rev. Lett. 35, 963 (1975).

${ }^{13}$ I. N. Goncharenko and I. Mirebeau, Europhys. Lett. 37, 633 (1997).

${ }^{14}$ H. Kepa, G. Springholz, T. M. Giebultowicz, K. I. Goldman, C. F. Majkrzak, P. Kacman, J. Blinowski, S. Holl, H. Krenn, and G. Bauer, Phys. Rev. B 68, 024419 (2003).

${ }^{15}$ J. C. Le Guillou and J. Zinn-Justin, Phys. Rev. B 21, 3976 (1980).

${ }^{16}$ E. Scheer, J. Wosnitza, H. v. Löhneysen, R. Kürsch, M. Lang, and F. Steglich, J. Magn. Magn. Mater. 104-107, 175 (1992).

${ }^{17}$ R. M. Mueller, U. Kobler, and K. Fisher, Eur. Phys. J. B 8, 207 (1999).

${ }^{18}$ G. Will, S. J. Pickart, H. A. Alperein, and R. Nathans, J. Phys. Chem. Solids 24, 1679 (1963).

${ }^{19}$ D. S. Rodbell, I. S. Jacobs, J. Owen, and E. A. Harris, Phys. Rev. Lett. 11, 10 (1963).

${ }^{20}$ C. P. Bean and D. S. Rodbell, Phys. Rev. 126, 104 (1962).

${ }^{21}$ U. Kobler, A. Hoser, H. A. Graf, M.-T. Fernandez-Diaz, K. Fischer, and T. Bruckel, Eur. Phys. J. B 8, 217 (1999). 